\title{
Las teorías de la organización: ¿Funcionan para explicar de manera integral a las organizaciones?
}

Pascuala Josefina Cárdenas Salazar ${ }^{1}$

\section{RESUMEN}

En el mundo administrativo han surgido teorías que intentan de dar respuesta a los problemas organizacionales, mismas que con diferentes enfoques que tratan de entender y describir los fenómenos que las afectan y como predecir futuros eventos, sin embargo, sus postulados podrían estar dejando de lado factores que inciden en el funcionamiento de una organización y tal vez no cumplan el objetivo para el que fueron creadas. En este ensayo se analizan brevemente los postulados de las distintas teorías de la organización se inicia el análisis con la teoría de la administración científica, luego se habla de la teoría clásica, posterior la teoría de las relaciones humanas, luego, la teoría de la burocracia, posteriormente se mencionará la teoría del comportamiento, se incluye la teoría de los sistemas cooperativos, después la de sistemas y finalmente la teoría de la contingencia. Se concluye que ninguna teoría contempla de manera integral las variables que explican su funcionamiento y se propone un estudio que de manera holística contemple todas las variables que inciden en el diseño de la organización, considerando también de manera integral todos los aspectos del hombre, considerando las distintas disciplinas del conocimiento.

Palabras claves: Teoría, organizaciones, administración, relaciones humanas, burocracia, sistemas

Recibido: 08 de mayo de 2016

Aceptado: 10 de junio de 2016

2 Estudiante de Maestría en Gestión Organizacional con enfoque en investigaicón en el Instituto Tecnológico de Sonora (ITSON). Correo Eletrónico: laejosefina@gmail.com 


\title{
Organization theories: Do they work to explain the nature of organizations in an integral way?
}

\begin{abstract}
In the administration world, some theories that intend to give an answer to the organizational problems, and that try to understand and describe the phenomena that affect them, have emerged. However, their postulates could omit factors that impact the functioning of organization, and may not fulfil the objective for which they were created. In this essay, the postulates of different organization theories are briefly analyzed. First, the theory of scientific administration is analyzed. Then, the classical theory, the theory of human relations, the theory of bureaucracy, the theory of human behavior, the theory of cooperative systems, and finally the theory of contingency are analyzed. It is concluded that none of the theories integrally addresses the variables that explain its functioning, and a study that holistically addresses them is proposed. This study should include the variables that influence in the organization, all aspects of the human being, and the different disciplines of knowledge.
\end{abstract}

Keywords: Theory, organization, administration, human relations, bureaucracy, systems 


\section{INTRODUCCIÓN}

Las organizaciones en la actualidad están inmersas en un ambiente dinámico, inestable, turbulento y cambiante y como menciona Hamel (2008), el ambiente que enfrentan en el siglo XXI, es más volátil que nunca, además, traen consigo grandes retos que requieren nuevas realidades, necesitan de acciones oportunas que les permitan prosperar y adaptarse. Cada vez hay más de éstas y se han convertido en una parte muy necesaria en la vida de las personas. A propósito de ello, Pfeffer (2000) menciona que se han multiplicado las organizaciones; no sólo hay más, sino cada vez son más grandes, cada vez, más organizadas y más complejas y por cierto, sus estructuras han sido poco estudiadas.

Es por eso que han surgido teorías con el propósito de dar respuesta a los problemas organizacionales, mismas que con diferentes enfoques que tratan de entender y describir los fenómenos que las afectan y como predecir futuros eventos. Sin embargo, estos supuestos pudieran estar dejando de lado factores que inciden en el funcionamiento de una organización y tal vez no cumplan el objetivo para el que fueron creadas. Es por eso que este ensayo tiene el propósito de analizar las teorías que se han dedicado al estudio de la organización y saber si ¿funcionan para explicar, de manera integral cómo funcionan las organizaciones?.

Para ello, se analizan brevemente los postulados de las distintas teorías de la organización se inicia el análisis con la teoría de la administración científica, luego se habla de la teoría clásica, posterior la teoría de las relaciones humanas, luego, la teoría de la burocracia, posteriormente se mencionará la teoría del comportamiento, luego la teoría de los sistemas cooperativos, después la de sistemas y como punto final la teoría de la contingencia. Asimismo para hablar de los aspectos que manera integral conforma una organización, se hará de forma general, ya que entrar especificar a detalle sería una lista muy extensa, por tanto, a manera general, se toman como punto de referencia las aportaciones de Bunge (1980), quien menciona que los factores que componen una organización son el ambiente, la estructura y los individuos miembros de la misma.

\section{FUNDAMENTOS DE LAS TEORÍAS DE LA ORGANIZACIÓN}

Conocer los fundamentos que tienen las distintas teorías podría ayudar a entender si los postulados que ofrecen sus representantes explican la naturaleza de las organizaciones y atiendan de manera integral todos los factores que inciden en el funcionamiento de las organizaciones.

\section{Teoría de la administracion científica}

El primer esfuerzo para atender a los problemas organizacionales es a partir de los trabajos de Taylor (1911) quien buscó darle solución a situaciones como prácticas de trabajo ineficaces, bajo rendimiento sistemático, bajo rendimiento natural, antagonismo entre trabajador y patrón, entre otros problemas y pretendió una máxima prosperidad para el patrón y para el trabajador, a través del máximo rendimiento del trabajador. Para ello implementa cuatro principios que consistieron en primer lugar en sistematizar los procesos, precisando que debía existir una mejor forma de hacer las cosas en cada organización, misma que debían seguir todos los empleados, the one best way.

Después de eso, implementó la selección científica de los trabajadores y un programa de adiestramiento. También dividió el trabajo y la responsabilidad entre la dirección y los trabajadores, supervisó y especializó a los trabajadores, con el thinking deparment se apropió del conocimiento, del saber hacer y con esto, según él subsana es discordia y logra una relación amorosa. Y por último, planteó hacer labor de convencimiento para que los trabajadores trabajaran cordialmente y llevar a cabo el control, para asegurarse de que todo el trabajo se hiciera de acuerdo con los principios de la ciencia. 
Después de los postulados expuestos, se podría decir que la teoría de la administración científica sólo atiende aspectos técnicos. Como puede apreciarse en Barba (2013), quien se refiere a dichos postulados como procesos y técnicas para lograr la eficiencia de la producción. También Ballina (2006), quien menciona que el propósito básico de la obra de Taylor era incrementar la productividad organizacional a través de eficientar y sistematizar los procesos. Barba (2010) define al taylorismo como movimientos eficientes y la reducción de pérdida de tiempo en las actividades, principios que regulan los procesos, menor responsabilidad para los trabajadores y sobretodo garantía en que en todo momento se hagan las cosas conforme las normas.

En esta postura teórica se dejaron de lado aspectos humanos, mismos que podrían ser útiles si la teoría debiera explicar también aspectos relacionados con los miembros de la organización. Al respecto, Indárraga (2006) comenta que la búsqueda de la eficiencia y la productividad, dejaron fuera la libertad de dominio que tenía el hombre sobre sí mismo y el mundo. Por su parte, Pacheco (2010) menciona que el fragmentar el trabajo a su máxima expresión, privó al hombre de su voluntad y conciencia. Ibarra y Montaño (1987) comentan que no se vio reflejada la máxima prosperidad para los trabajadores en los mecanismos correctivos, ni en salarios disfrazados de bienestar económicos. Por su parte, Gallardo y Zarur (2010) mencionan que esta forma de trabajo dejó al trabajador sin identidad.

Si la teoría de la organización no funciona para explicar a la organización, ¿por qué sigue vigente? Respecto al tema, Hernández y Ramírez (2010) comentan que los ideales y pensamiento administrativo de Taylor siguen latentes y ha dejado huella, pues muchos comparten su gusto por la medición del tiempo, disciplina, rendimiento, eficiencia y por encontrar la mejor forma de llevar a cabo una tarea; las ideas de Taylor se extendieron a varios países ejemplo de ello Inglaterra, en el cual dieron gran relevancia al estudio de tiempos y movimientos.
Otra razón de su vigencia podría ser la misma influencia de los postulados en la implementación de nueva tecnología, por ejemplo, Quiroz (2010) menciona que a partir de la llegada de inversión extranjera en la industria automotriz de 1974 a 1982, la influencia de los postulados de Taylor, venían implícitos en la instalación y equipos en sus formas de organización y gestión de la producción, tal como se evidenció en las investigaciones que se realizaron a las empresas Chrysler de México, Ford Company, S.A., General Motors y Nissan Mexicana; aplicaban técnicas de control de tiempos y movimiento y también resultaban conflictos organizacionales en una respuesta a la resistencia al cambio, como en todos los lugares en donde se aplican métodos, procesos y sistemas de trabajo de Taylor.

La filosofía de Taylor sigue siendo aplicada en las organizaciones actuales, aunque según Pacheco (2010) solo retoman de la postura lo que les conviene, se orientan generalmente a los procesos productivos, organización y medición del tiempo, pero no implementan otras acciones que también eran parte del mismo ideal, tal como un salario justo al trabajador, por tanto, emplean de manera mutilada el sistema de trabajo Tayloriano. Hernández y Ramírez (2010) mencionan que los modelos de control actuales son semejantes al control operativo de Taylor, de tal suerte, comenta Hamel (2008) que Taylor se llevaría una sorpresa si fuera testigo de que los administradores modernos se obsesionan con sus postulados a más de 100 años.

Podría comentarse por tanto, que Taylor (1911) logró cambios importantes respecto de la situación que se propuso resolver; su objetivo de elevar la eficiencia y maximizar la productividad, una sistematización del trabajo, control y apropiación absoluta en el diseño de los procesos, sin embargo, dejó fuera aspectos que podrían incidir en el funcionamiento de la organización, tales como el ambiente y sobre todo, respecto al trato que le dio al hombre como un objeto, como una herramienta, como una pieza más del engranaje que, 
como puede reflejarse en varios críticos no trajo tan buenos resultados.

\section{Teoría clásica}

El segundo esfuerzo es la identificación de principios, la estructura y el funcionamiento de la organización; se refiere a la teoría clásica de Fayol (1916). En ella se misma que identifica en la organización seis operaciones o funciones, las técnicas, comerciales, financieras, de seguridad, contabilidad y administrativas; proponiendo que de todas ellas, las operaciones administrativas son a las que se debe estudiar con detenimiento y las enuncia como prever, organizar, dirigir, coordinar y controlar. Asimismo, proyecta los principios generales que deberían regir la administración. Precisando de una vez que según Parker y Ritson (2005) operaciones y los principios administrativos conforman el andamiaje de la teoría de Fayol.

Aunque los autores mencionados con anterioridad mencionan que Fayol (1916) de cierta manera previó las relaciones humanas al dar importancia al individuo, él mismo, en su libro de la Administración industrial y general, menciona que no debía debilitarse el engranaje administrativo, de esta manera, cuando un organismo obedece al impulso del jefe, las partes unidas se mueven juntas hacia la misma dirección, cabe agregar que de las personas solo se preocupa que cumplieran los requisito, más que ocuparse de sus necesidades físicas o sociales.

Por las consideraciones anteriores se podría decir que Fayol dejó también aspectos que caracterizan a la organización, por tanto, no podría considerarse integral si se ve desde este punto de vista meramente funcional, sin embargo, si no funciona para explicar de manera integral cómo funciona una organización, ¿por qué continúa vigente? Una explicación de que los postulados subsisten actualmente es su influencia en nuevos conceptos, aunque Rivas (2007) menciona que con frecuencia se usa el modelo funcional en las organizaciones, aunque con malos resultados.
Otra de las explicaciones de su vigencia es la de Fells (2000), quien comenta que su teoría está inmersa o incluida en otros enfoques más modernos; algunos principios, por ejemplo, el orden, en el concepto de Just in Time (justo a tiempo), la división del trabajo, en enfoques avanzados en la línea de ensamble y calidad, en los mecanismos de producción, el espíritu de cuerpos en los círculos de calidad y sesiones de ejercicio y la iniciativa en la toma de decisiones de niveles inferiores. Por su parte Macias y Vidal (2002) comparten que esta teoría tiene un enfoque de sistema cerrado, no toma en cuenta los factores ambientales ni su influencia y además no explica de manera correcta el comportamiento de las personas y sus principios y fundamentos son contradictorios.

\section{Teoría de las relaciones humanas}

El concepto del trabajador se transformó a partir de la teoría de las relaciones humanas de Mayo (1946), ésta, resultó después de una serie de investigaciones en donde primero estudió la fatiga y monotonía de los trabajadores, entre otros estudios relacionados con el bajo desempeño laboral. Sin embargo, fue a partir de los resultados de la investigación que realizó en la Hawthorne, en la Western Electric Company, de 1924 a 1932, durante una serie de fases que se fueron modificando los beneficios a seis obreras quienes trabajaban en el ensamble dispositivos, a quienes se les vigiló y se les modificaron las condiciones laborales sorprendiendo con su alto desempeño laboral a pesar de la suspensión de los beneficios; se descubre la influencia de la organización informal, a la que se refieren Roethlisberger y Dickson (1934) como una variable que podría emplearse para favorecer el rendimiento de los trabajadores.

Se puede apreciar cierta inclinación hacia las necesidades sociales del individuo, como lo comenta Saracheck (1968) se le da importancia como ser y como hombre social, sólo que con el objetivo de alcanzar un buen rendimiento productivo, aunque contrario a lo que dice Schwartzman (1993), que se cambió el concepto 
del hombre con necesidades e intereses sociales y psicológicos en oposición al hombre economía de Taylor. Por su parte, Cooper (1962) menciona que tanto Taylor como Mayo pretendían establecer y mantener la cooperación en la industria; mientras que el primero fue el padre de la gerencia científica, y que aumentó la producción industrial para sistematizarla el segundo fue el padre de la industria de las relaciones humanas y que incrementó la producción para humanizarla.

Ante la situación planteada se pueden observar elementos distintos a partir de que surge esta teoría, todos relacionados con la organización informal; expectativas de los empleados, necesidades psicológicas y redes de comunicación, como lo menciona Schwartzman (1993) que se cambió el concepto del hombre con necesidades e intereses sociales y psicológicos en oposición al hombre economía de Taylor. O como menciona Friedmann (1956), que hay un claro avance que según se traduce en integración intelectual, afectiva y social. Aunque se reconoce un cambio en los factores que incluye, deja de lado otros aspectos tales como el ambiente, las relaciones, y la mismo individuo, quien aún sigue siendo considerado un instrumento, sólo que ahora es una herramienta-social.

\section{Teoría de la burocracia}

Esta teoría, a diferencia de las anteriores, no fue hecha como un modelo para entender a las organizaciones, sino, para comprender cómo interpretan la realidad las personas, lo que podría presumir de antemano dificultad conocer cómo funcionan las organizaciones a través de ella. Como se puede apreciar en sus reflexiones, Weber (1964) crea un instrumento que sirve para comprender e interpretar los significados que las personas le confieren a sus propias acciones, no para obtener la máxima eficiencia ni para usarlo como modelo.

El mismo autor se refiere a su tipo ideal porque no es real, sólo exagera los rasgos y hace comparaciones, determinando así el grado de desviación, así se podría explicar por qué el hombre se comporta de cierta manera ya sea si apela a la razón, a la emoción o a la tradición. Soportando lo anterior, Mouzelis (1991) comenta que este tipo ideal no fue hecho para ser aprobado empíricamente, sólo para comprender fenómenos sociales, $y$, aunque algunos rasgos exagerados se pueden observar en menor o mayor grado en las organizaciones existentes, el tipo ideal de burocracia en su pureza, no se encuentra nunca en la realidad.

Se considera que la teoría de la burocracia es una postura teórica que ha sido más criticada por la mala interpretación y fallas en la aplicación por la mala interpretación. Se cita por ejemplo a Merton (2003) quien critica las características de la burocracia que observa en algunas de las organizaciones como si se tratara de algo que Weber haya pretendido. En el mismo entendido se encuentran los trabajos de Crozier (1969) quien encuentra total incompatibilidad entre los objetivos de las organizaciones estudiadas y un sistema burocrático, criticando así la rigidez de la estructura, los mecanismos de control, las normas y las reglas. De una forma que fue empleado como un modelo y no como el mismo autor lo propuso.

De acuerdo con los razonamientos que se han venido realizando se puede decir que la burocracia ha sido adoptada como un modelo, sin serlo, y con malos resultados. Sin embargo, si la teoría de la burocracia no funciona para explicar de manera integral a la organización, ¿por qué sigue vigente?, Rivas (2007), menciona que la teoría burocrática es una de las teorías que se emplean tal vez porque es de las que más se conoce aunque tenga malos resultados.

El mismo Weber (2005), menciona que una organización burocrática bien desarrollada, constituye una de las organizaciones de más difícil destrucción y de sustituir, el funcionario no puede desprenderse de la organización y los gobernados no pueden prescindir del aparato de dominio; cualquiera podría adaptarse y es fácil de controlar. Asimismo, Hamel (2008) menciona que los rasgos de precisión, estabilidad, disciplina, 
entre otros, continúan vigentes en la administración moderna, principios que rigen principalmente los sectores públicos en el mundo.

De las reflexiones anteriores, se deduce que la teoría difícilmente podría funcionar para explicar cómo funciona una organización, primero por no tratarse de un modelo, luego, por dejar fuera aspectos que inciden en la organización como el ambiente y los individuos, ya que como lo menciona Mouzelis (1991) que una organización entre más burocrática y racional, los individuos se reducirán a simples piezas de un mecanismo, ignorantes de un significado y de una interpretación de lo que hacen. Además, al parecer según Parson (citado en Mouzelis 1991) deja fuera también aspectos técnicos e institucionales para centrarse sólo a la parte burocrática.

Por lo tanto, el concepto de organización tiene un sentido nuevo, pues en las anteriores teorías, solo importaban las tareas, las funciones o las relaciones de las personas, aunque cabe mencionar que en esta teoría, no incluye los aspectos que en las anteriores, como las relaciones informales, las funciones, los procesos, las técnicas; mucho menos el ambiente y las variables integrales del individuo y se enfoca sólo a la estructura de la organización.

\section{Teoría del comportamiento}

La siguiente teoría que intenta explicar cómo funcionan las organizaciones es desde un enfoque del comportamiento, para Simon (1982) la toma de decisiones es el punto medular en el estudio del comportamiento individual por su trascendencia en el comportamiento grupal. Quien toma la decisión, desde su ámbito jerárquico, debe tener una visión panorámica de las alternativas, considerar todas las consecuencias y realizar una evaluación comparativa, sin embargo, el conocimiento se considera imperfecto ya que solo se sabe parte de las condiciones que rodean su elección, como lo menciona March (1994) quienes toman las decisiones tienen serias limitaciones de atención, memoria, comprensión y comunicación. En March (1989) se explica que la empresa comercial es una organización que requiere de la elaboración e implementación de decisiones dentro del mercado económico.

En cuanto a su vigencia en las organizaciones podría ser la influencia que reciben los nuevos administradores en sus universidades, luego, ellos en sus empresas; como comenta Ramió y Ballart (1993) que una vez que se dieron a conocer teorías como la del comportamiento y otras de psicología, el campo de comportamiento administrativo empezó a establecerse, así en las escuelas de estados unidos, en sus programas de licenciatura en áreas de administración de empresas, administración pública o administración hospitalaria tienen un curso obligatorio de comportamiento organizativo.

Como se observa en la teoría del comportamiento, no se puede explicar el fenómeno organizacional solo a través del comportamiento. Se podría decir, que al igual que en el caso de las anteriores, no incluye todos los aspectos que inciden en el funcionamiento de una organización, sino, se enfoca a ciertos factores, en este caso, a la toma de decisiones que repercute en el comportamiento individual y grupal, el mercado y la política; mas no incluye los aspectos de las anteriores teorías, ni considera todos los factores que influyen en el diseño de la organización.

\section{Teoría de sistemas cooperativos}

Otra de las teorías que han surgido para el estudio de las organizaciones es la de sistemas cooperativos, Barnard (1938) considera a las organizaciones como sistemas cooperativos, es decir, organizaciones cuyos miembros comparten esfuerzos para lograr los propósitos de la organización. El autor también expone el trabajo de los directivos como canales por medio de los cuales la comunicación fluye, a la autoridad como una manera en que los trabajadores deciden aceptar la orden. 
También resalta la importancia de la individualidad del hombre y sus factores psicológicos, biológicos y físicos aunque reconoce que las personas como participantes en los sistemas cooperativos específicos se consideran en sus aspectos puramente funcionales y de cooperación. El autor considera relevante a los grupos informales dentro de las formales, estos últimos son considerados un tipo de cooperación humana que es consciente, deliberada y que está encaminada a un fin.

Significa entonces que en los sistemas cooperativos existe un nuevo elemento como la importancia que se le da a los individuos y a los incentivos para que los que dirigen las organizaciones cooperen de manera adecuada para el logro de los objetivos, se considera que se apoya en la teoría del comportamiento, pues retoma conceptos solo que los enfoca a las funciones del ejecutivo, los grupos formales son importantes, pero también incluye los grupos informales. Se puede decir que tampoco se puede explicar de manera integral a la organización a través de sistemas cooperativos, pues no se puede explicar de manera integral a la organización si solo se considera su diseño a través de la coordinación de la misma y si solo se enfoca a las funciones del directivo.

\section{Teoría de sistemas}

La teoría de sistemas intenta ser integradora pues su principal representante Bertalanfy (1968), contempla varios aspectos que no habían sido contemplados hasta el momento, el sistema es definido por el conjunto ordenado de elementos que interaccionan entre sí para el logro de determinado objetivo, de esta manera la variabilidad de sus partes incide en los demás y por tanto, influye en el conjunto y su complejidad depende de la cantidad de elementos y relaciones en que interactúan. Kast y Rosenzweig (1993) consideran elementos del sistema, al modelo de entrada, salida, transformación, entropía negativa, estado estable, equilibrio dinámico y homeóstasis y retroalimentación de los sistemas abiertos. Existen sistemas dentro de sistemas, los sistemas son abiertos y la estructura determina el funcionamiento de la organización.

Una de las situaciones que podrían limitar que se explique el funcionamiento de una organización a través de esta teoría es por tratar de explicarla como un organismo vivo, Bertalanffy (1968) se apoya de la estructura de un organismo viviente, para explicar lo cómo podría explicarse un sistema abierto, el cual que se mantiene en continua incorporación y eliminación de materia, construyendo y demoliendo componentes, sin alcanzar, mientras la vida dure, un estado de equilibrio. Katz y Khan (1972) comentan que un sistema es abierto cuando no puede ser definido por un conjunto estático de relaciones causa-efecto organizacional, el entorno y medio ambiente interno, así como las interrelaciones entre los sistemas y subsistemas.

La teoría de sistemas aporta nuevos elementos como considerar las interrelaciones de la organización con el medio ambiente, por considerar que existen subsistemas dentro del sistema y considerar que el funcionamiento depende de la estructura de la organización. Evidentemente integra más elementos para el estudio de la organización, sin embargo, no establece que tipo de relaciones establece, cómo se relaciona, cómo influye o cómo afecta. Además, no se puede explicar a partir de un organismo vivo, que de por sí es complejo, a una organización, pues la última posee complejidades distintas y funcionamientos diferentes. La visión del individuo también cambia, ahora forma parte de todo un sistema, sin embargo, sigue siendo un instrumento, ahora una herramientaorgánica, es decir, no se considera a manera integral los aspectos del hombre.

\section{Teoría de contingencia}

Como punto final, se tiene la teoría de la contingencia, la cual, está enfocada en el medio ambiente y su impacto como factor determinante de la estructura y funcionamiento de la organización. Para Gunther (2006) la hipótesis de la contingencia es que las 
organizaciones que se ajustan sus estructuras dependiendo de su ambiente, supera el rendimiento de las organizaciones que no modifican su estructura. Para Crozier y Friedberg (1990) la teoría de la contingencia estructural se enfoca a inventariar y describir las dimensiones de la dependencia que se da entre la organización y el medio ambiente externo, además de medir el impacto respectivo sobre la estructura y funcionamiento.

Como puede apreciarse dentro de los resultados de sus experimentos de Woodward (1975), encontró que no hay una mejor forma de hacer las cosas que aplique a todas las situaciones, como la teoría clásica lo propone y además, que existe una relación entre el desempeño y el tipo de tecnología empleado en los procesos productivos. Asimismo Pugh et al. (1973) quienes después de sus estudios en Inglaterra dieron un enfoque multidimensional a la estructura organizacional: especialización, estandarización, formalización, centralización, configuración, y flexibilidad; cinco fueron operacionalizadas y se obtuvieron 64 escalas para medir los aspectos de las dimensiones. Dicha estructura puede variar en cada una de las dimensiones; esto permitía elaborar perfiles característicos de las organizaciones e identificar diferencias estructurales, también se podría realizar enfoque multivariado de causalidad.

Otra investigación que soporta esta teoría, misma que pretende demostrar que el entorno afecta la estructura de las organizaciones y su funcionamiento, es la realizada por de Burns y Stalker (citados en Croazier y Friedberg, 1990, quienes clasifican dos tipos de organización mediante sus estudios realizados en 20 empresas en Gran Bretaña: el modelo mecánico y el modelo orgánico; el entorno afecta a la organización dependiendo del tipo que sea. Para conocer cuál sería un factor que influye en los resultados alcanzados por una organización, Emery y Trist (citados en Croazier y Friedberg, 1990) realizan una clasificación del contexto en que las organizaciones se encuentran, tranquilo y disperso; tranquilo y agrupado; perturbado y reactivo y por último; turbulento.

Se encuentran más coincidencias de que ciertas condiciones del ambiente afecta otros factores organizacionales y de ello depende el resultado, Lawrence y Lorsh (citados en Croazier y Friedberg, 1990) quienes comentan que existe relación entre las variables externas, el estado interno de la organización y los procedimientos para resolver conflictos, además, en la manera que se estos factores se ajusten, será el resultado que se obtenga. De ellos se acepta la premisa fundamental de la teoría, de que no existe una guía universal, y que la integración y la diferenciación se logran dependiendo de las condiciones del medio y de la situación a resolver, una situación contingente para la organización.

En la teoría de contingencia los conceptos nuevos que trata a diferencia de la teoría de sistemas es que la estructura y el funcionamiento de la organización no solo interrelacionan con el medio ambiente, sino que el medio ambiente determinan la estructura y funcionamiento de la organización. Factores como tamaño de la organización, el medio ambiente y la tecnología son variables independientes que afectan las variables dependientes dentro de la organización, como las dimensiones de la estructura.

Esta teoría, aunque incorpora bastantes aspectos de la organización respecto al individuo, el ambiente y la estructura, así como sus interrelaciones, considera diferentes aspectos de la organización que el resto de las teorías analizadas. Se observa que deja fuera elementos variables que pudieran estar afectando a la organización, como el origen, historia, localización, así como no considera variables dependientes que se ven influenciadas tales como los procesos o las relaciones informales y otras que podrían requerir de un enfoque central. 


\section{CONCLUSIONES}

Para concluir, después de analizar las teorías sus postulados y su vigencia, podría decirse ninguna integra todos los factores que inciden en el funcionamiento de una organización, y cada teoría posee distintas características que intentan ayudar a entender el diseño de una organización, es decir, cada una enfoca su atención a distintos factores que inciden en el los fenómenos organizacionales; en ese entendido, se podría decir que no hay punto de comparación entre ellas, tal vez porque cada una ha surgido en diferentes épocas, atendiendo a distintos problemas.

Por ejemplo, Taylor centra su atención a los procesos, por su parte, el trabajo de Fayol es relacionada con el de Taylor, pero como lo mencionan Parker y Ritson (2005), tal vez sólo por ser contemporáneos, porque se les adjudica una base administrativa autoritaria y el empleo de leyes inmutables o inflexibles. No obstante, también deja fuera elementos necesarios para entender integralmente el funcionamiento de las organizaciones. La teoría de relaciones humanas, tampoco es la excepción, Schwartzman (1993) comenta que las necesidades psicológicas y los intereses sociales son tomadas en cuenta en esta teoría y Friedmann (1956) dice que las necesidades afectivas y sociales también son consideradas. Sin embargo, se podría concluir que tampoco es suficiente para explicar el funcionamiento integral de las organizaciones.

Se considera que la teoría de la burocracia es una postura teórica que ha sido más criticada por las fallas en la aplicación debido a una mala interpretación, que por la esencia de sus propios postulados. Weber (1964) construyó un instrumento analítico con la pretensión de interpretar los fenómenos sociales. Es una construcción social, así que esto presume, de antemano, dificultad para implementar esa herramienta en alguna estructura social. En la teoría del comportamiento aunque se incluyen otros factores como el mercado, la política, y el proceso de la toma de decisiones y se reconoce que el que toma las decisiones es una persona, no posee todos los elementos que intervienen en el funcionamiento, por ello, tampoco explica cómo funciona una organización.

La teoría de sistemas cooperativos solo que los enfoca a las funciones del ejecutivo, la forma de incentivarlo, entre otras relacionadas con la cooperación, lo cual tampoco posee lo necesario para entender de forma total a las organizaciones. La teoría de sistemas, aunque aporta nuevos elementos como considerar las interrelaciones de la organización con el medio ambiente, por considerar que existen subsistemas dentro del sistema y considerar que el funcionamiento depende de la estructura de la organización e teoría intenta ser integradora o con visión holística, se considera que es muy general y poco práctica por considerar elementos de un organismo vivo para explicar las formas complejas de interrelaciones entre los individuos, así como entre organizaciones, y por ello, se podría decir que no es suficiente para entender cómo funcionan las organizaciones.

En la teoría de contingencia se podría decir que los conceptos nuevos que trata es que a diferencia de la teoría de sistemas, la estructura y el funcionamiento de la organización no solo interrelacionan con el medio ambiente, sino que el medio ambiente determina la estructura y funcionamiento de la organización. Factores como tamaño de la organización, el medio ambiente y la tecnología son variables independientes que afectan las variables dependientes dentro de la organización, las dimensiones de la estructura. Sin embargo, como lo menciona Croazier y Friedberg (1990) que la teoría se podría considerar como un sesgo de la teoría clásica de las organizaciones y además determinista y unilateral, al considerar que es el medio ambiente el que determina la estructura y funcionamiento de la organización.

Lo cual lleva a concluir que la teoría de contingencia tampoco se podría aceptar como completa para entender de manera integral a las organizaciones, tal vez podría ser de mayor utilidad para resolver los problemas específicos de situaciones especiales que se adaptan 
a las características y requerimientos propios de cada organización pero deja fuera variables independientes que pudieran estar afectando a la organización, como el origen, historia, localización, así como no considera variables dependientes que se ven influenciadas tales como los procesos o las relaciones informales.

Por tanto, se observa que las teorías revisadas no poseen los elementos necesarios para la interpretación holística de la realidad organizacional, de alguna manera deja en evidencia que sus formas no explican una realidad actual, al menos no de una manera integral, algunas tal vez a resolver situaciones específicas, pero difícilmente a predecir fenómenos. No se deja de reconocer que los diferentes modelos fueron creados en un momento histórico determinado que encajan poco con las condiciones sociales actuales, ya que el contexto en el cual surgieron, las prioridades y problemas clave eran otros, la época actual tiene sus propios retos y sus propios problemas, cuyas soluciones tendrán que emanar de otros modelos.

Respecto al ¿por qué se siguen empleando modelos teóricos que tienen más de cien años desde su creación? Y como se aprecia en algunos estudios en que se evidencia la existencia de algunos modelos de organización, la pregunta sigue sin contestarse, pero se propone poner a prueba las teorías para que existan nuevas evidencias que permitan a los administradores o nuevos dirigentes tener herramientas y bases sólidas de que el conocimiento y técnicas que postulan las teorías, es conveniente para entender cómo funcionan las organizaciones.

También se propone que los programas educativos contengan la gama completa de los modelos teóricos, y que en lugar de que los nuevos administradores adopten modas, pudieran recibir dentro de sus instituciones un entrenamiento que logre en ellos la habilidad de gestionar organizaciones, de profundizar en los problemas, de diseñar nuevas estructuras organizaciones, de analizar de manera crítica las situaciones y de llegar a conclusiones relevantes para la organización, así como dejar de adoptar modelos que podrían no encajar con las necesidades específicas de las organizaciones. Los estudiantes saldrían preparados para atender las necesidades apremiantes de las organizaciones para las que se trabajarán.

En base en análisis de las teorías y a las observaciones a algunas investigaciones se puede observar que se ve al hombre como un objeto, es decir, como un medio para alcanzar los fines organizacionales ya sea como una herramienta mecánica, funcional, cooperativa, ambiental u orgánica. Por ello podría concluirse que es urgente una visión más integral del hombre en las organizaciones, que considere sus aspectos biológicos, psicológicos, sociales e históricos.

Como se observó, las teorías se han enfocado de manera asilada a distintas variables de la organización, tales como procesos, tareas, funciones, a la estructura formal, a las relaciones informales, al comportamiento y su motivación, a la interrelación con el medio ambiente etc., esto podría ser una limitante para entender y atender a las necesidades integrales de una organización, sin embargo, se reconoce la acumulación del conocimiento que postula cada teoría enfocada a diferentes aspectos organizacionales.

Por lo tanto, se concluye también, que las teorías podrían no ser excluyentes y tal vez complementarse unas con otras para interpretar de manera holística la naturaleza social de la organización, ya que todas consideran puntos de vista diversos, todas con origen en distintas áreas de la ciencia. Esa diversidad podría combinarse para comprender los distintos aspectos de un contexto de una manera interdisciplinaria y se podría conseguir una visión integral de la realidad organizacional.

Al respeto, se propone un estudio integral de la organización, que analice y reflexione todas las variables que intervienen en el funcionamiento de una organización, procesos, relaciones sociales, estructura, estilos de dirección, el ambiente (social, legal, político, 
económico, natural, moral, profesional, cultura) y a los actores (trabajadores, sociedad, proveedores, clientes y competencia). En el mismo que se incluya un análisis integral multidisciplinario que se reflexione desde las distintas áreas del conocimiento (hasta ahora contempladas, la ingeniería, la psicología, economía, administración, biología).

Se requiere de urgencia, romper con paradigmas, que las instituciones encargadas de formar nuevos dirigentes no se casen algún modelo, que quienes administren las organizaciones no se adopten una idea como si se tratara de la panacea, que quienes se encarguen de investigar puedan llegar a descubrir nuevas formas, nuevos modelos que puedan adaptarse a las necesidades integrales de las organizaciones, pues las teorías de hace 100 años, fueron realizadas para resolver situaciones de hace cien años y no obstante, las organizaciones actuales requieren de visiones que permitan entender sus circunstancias reales, vigentes y presentes; que por lógica no podrían atenderse con posturas del pasado. Aunque aquí no se propone dejar en el olvido dichas posturas, sino, utilizarlas como marco de referencia, como antecedente o como marco teórico. Como dice Kuhn, (2011) para que haya progreso debe haber un cambio de supuestos teóricos, un cambio del patrón usual del proceso de la ciencia, un cambio de paradigma.

El tipo de investigación que pudiera sugerirse para futuros estudios respecto a las teorías analizadas depende de qué se quiere investigar, si se requiere de una investigación profunda, la investigación no puede simplificarse a un cuestionario (instrumento) se propone una investigación cualitativa, aunque por otro lado, la información obtenida sería de un lugar, con problemas y situaciones específicas y el conocimiento que emana de la investigación difícilmente podría generalizarse, como es el propósito de las teorías. En general, la cualitativa cuando se requiera entender, interpretar y explorar problemas sociales o humanos y la investigación cuantitativa cuando se requiere poner a prueba las teorías, buscar correlaciones entre variables, predecir fenómenos o generalizar resultados.

\section{REFERENCIAS BIBLIOGRÁFICAS}

Ballina, F. (2006). Diferencias en la administración premoderna, moderna y posmoderna: propuestas para un debate en Latinoamérica. Problemas de Desarrollo, 37 (144), 222-233.

Barba A. (2010). Frederick Winslow Taylor y la administración científica: contexto, realidad y mitos. Gestión y estrategia (38).

Barba A. (2013). Administración, teoría de la organización y estudios organizacionales: tres campos de conocimiento, tres identidades. Gestión y Estrategia, 44.

Bertalanfy, L. (1968). Teoría general de los sistemas. Fondo de Cultura Económica. Mexico, Madrid, Buenos Aires.

Barnard, C. (1938). The functions of the executive.

Bunge, M. (1980). Status epistemológico de la administración. Scarano, R.(comp.), op. cit. Cap, 17.

Crozier, M. (1969). El fenómeno burocrático, ensayo sobre las tendencias burocráticas de los sistemas de organización modernos y sus relaciones con el sistema social y cultural. Buenos Aires: Amorrortu Editores.

Crozier, M. \& Friedberg, E. (1990). El actor y el sistema. Las restricciones de la acción colectiva, pp. 109-133. Alianza Editorial mexicana.

Fayol, H. (1916). Administración Industrialy Gerencia. México: Herrero Hermanos.

Fells, M.J. (2000). Fayol stands the test of time. Journal of Management History, 6 (8), 345-360.

Friedmann, G. (1956). Problemas humanos del maquinismo industrial. Buenos Aires: Editorial Sudamericana

Gallardo, A., \& Zarur A. E. (2010). Los nuevos usos de Taylor en el control del comportamiento humano. Gestión y Estrategia, (38), 97-107.

Hamel, G. (2008). The future of management. Human 
Resource Management International Digest, 16(6).

Hernández, A., \& Ramírez, G. (2010). El control administrativo de F.W. Taylor, cien años después. Gestión y Estrategia, (38), 61-73.

Ibarra E., y Montaño L. (1987). Mito y poder en las organizaciones: un análisis crítico de la teoría de la organización. (2da. ed). México: Editorial Trillas.

Indárraga, D. A. M. (2006). El sujeto humano en la administración: una mirada crítica. Cuadernos de Administración, 19 (32), 135-156.

Kast, F. E., \& Rosenzweig, J. E. (1993). Administración en las organizaciones: enfoque de sistemas y de contingencias. McGraw-Hill.

Katz, D. y Khan, R. (1972). Psicología social en las organizaciones. Atlas, Sao Paulo.

Kuhn, T. S. (2011). La estructura de las revoluciones científicas. Fondo de cultura económica.

Macias, A. M., \& Vidal, A. A. (2002). Evolución de la teoría Administrativa. Una visión desde la Psicología Organizacional.

Mayo, E. (1946). Problemas humanos de una civilización industrial. (2da. ed). Buenos Aires, Argentina: Ediciones Nueva Visión.

March, J. G. (1989). Decisions and organizations. Blackwell.

Mouzelis, N. P. (1991). Organización y burocracia: un análisis de las teorías modernas sobre organizaciones sociales. Barcelona: Ediciones Península.

Merton, R. K. (2003). Teoría y estructura sociales. México: Fondo de Cultura Económica. Páginas 275-286.

Pacheco, A. A. (2010). El Taylorismo: implicaciones técnicas y políticas, a cien años de distancia. (Spanish). Gestión y Estrategia, (38), 89-96.

Parker, L.D. \& Ritson, P.A. (2005). Revisiting Fayol: Anticipating contemporary management. British Journal of Management, 16, 175-194.

Pfeffer, J. (2000). Nuevos rumbos en la teoría de la organización: problemas y posibilidades. México DF: Oxford University Press.

Pugh, D. S. et al. (1973). Dimension of Organization Structure. En H. Wolf (Ed.), Comparative Organizations, (pp. 441-470). Westmead, Farborough, Hants, England: Saxon House.

Quiroz, J.O. (2010). Taylorismo, fordismo y administración científica en la industria automotriz. Gestión y Estrategia. 38, 75-87.

Ramió,C.\&Ballart,X.(1993). Teoría dela organización. INAP. Recuperado de https://books.google.com. $\mathrm{mx} /$ books?hl=es\&lr=\&id=gik $7 \mathrm{wftCT} 64 \mathrm{C} \&$ oi $=\mathrm{f}$ nd\&pg $=$ PA $11 \&$ ots $=$ ZWCqQlu-bl\&sig $=8 \mathrm{gY} 5-\mathrm{A}$ IeAf2RjeS5RKbrkcx50s\#v=onepage \&q\&f=false

Rivas, L. A. (2007). Mapa de las teorías de la organización: una orientación para empresas. GacetaIde@s CONCYTEG, 2(23), 30.

Roethlisberger, F. J., \& Dickson, W. J. (1934). Management and the worker: Technical vs. social organization in an industrial plant (No. 9). Harvard University.

Sarachek, B. (1968). Elton Mayo's social psychology and human relations. Academy of Management Journal, 1 (2), 189-197.

Schwartzman, H. B. (1993). Ethnography in organizations. Qualitative research methods, (27), $1-26$.

Simon, H. A. (1982). El Comportamiento administrativo. Estudio de los procesos decisorios en la organización.

Taylor, F. (1911). The principles of scientific management. New York \& London: Harper Brothers.

Weber, M. (2005) ¿Qué es la burocracia? México: Ediciones Coyoacán.

Weber, M. (1964). Economía y Sociedad. México: Fondo de Cultura Económica.

Woodward, J. (1975), 'Management and Technology". En D. S. Pugh (Ed.), Organization Theory, (pp. 5671). 